\title{
LATTICE PACKING IN THE PLANE WITHOUT CROSSING ARCS
}

\section{LEONARD TORNHEIM ${ }^{1}$}

Introduction. We first review some definitions and results of Chalk and Rogers. ${ }^{2}$ If $S$ and $T$ are two sets of points in Euclidean $n$-space, then $S+T$ will denote the set of all points $s+t$ where $s$ is in $S$ and $t$ is in $T$, while $S-T$ is composed of all $s-t$. The point set sum will be denoted by $S \cup T$ and the intersection by $S \cap T$. Let $\Lambda$ be a lattice; then $S+\Lambda$ is a lattice packing if no two sets $S+\lambda$ and $S+\lambda^{\prime}$, with $\lambda$ and $\lambda^{\prime}$ distinct points in $\Lambda$, have a common point in their interiors. Let $D(S)$ be the set of all $s-s^{\prime}$ where $s$ and $s^{\prime}$ are points in the interior of $S$. Chalk and Rogers have shown that $S+\Lambda$ is a lattice packing if and only if the lattice $\Lambda$ is admissible for $D(S)$, i.e., has no point in the interior of $D(S)$ except possibly the origin $O$. If $S$ is convex this criterion reduces to a result of Minkowski, ${ }^{8}$ well known especially when $S$ is symmetric. For, if $S$ is also open, then $D(S)=S-S=S+S$ $=2 S$, this being the set $S$ expanded by a factor 2 .

From now on we assume that all point sets lie in the plane. We wish to provide a similar criterion for the situation in which no arc of $S+\lambda$ crosses an arc of $S+\lambda^{\prime}$. Then $S+\Lambda$ is called a lattice packing without crossing arcs, and is in particular a lattice packing.

Since $D(S)$ omits from consideration all arcs of $S$ not in the interior of $S$, it is to be expected that it will be of no use for our purpose. Our criterion will refer instead to the set $E(S)$ defined as $S-S$. Clearly $E(S)$ is symmetric. It is easy to see that $E(S)$ is generated by translating $S$ such that always one of its points is at $O$. If $E(S)$ is a circle and its interior then $S$ is a figure of constant breadth and conversely.

If $S$ is the square $0 \leqq x \leqq 1,0 \leqq y \leqq 1$, and $T$ consists of three sides of this square, omitting the side on the $y$-axis, then $E(S)=E(T)$. Yet there are lattices which provide lattice packings without crossing arcs for $T$ but which do not do so for $S$; e.g., the lattice generated by $(1 / 2,0)$ and $(0,1)$. The condition we obtain will involve local properties of $E(S)$ arising from $S$.

Presented to the Society, September 2, 1949; received by the editors March 26, 1952 and, in revised form, January 22, 1953.

1 This work was done under Contract N8-ONR-71400, Office of Naval Research.

$2 \mathrm{~J}$. H. H. Chalk and C. A. Rogers, The critical determinant of a convex cylinder, J. London Math. Soc. vol. 23 (1948) pp. 178-187; see pp. 186-187.

${ }^{8} \mathrm{H}$. Minkowski, Dichteste gitterformige Lagerung kongruenter Körper, Nachr. Akad. Wiss. Göttingen. Math.-Phys. Kl., 1904, pp. 311-355; see p. 313. 
We first discuss the crossing of arcs in general and obtain criteria applicable to our problem.

1. Crossing arcs. Denote by $e(A)$ the point set consisting of the two end points of an arc $A$.

Definition. Let $A, B$ be arcs with a common subarc $M$. Suppose that for every neighborhood $V \supset M$ there are arcs $A^{\prime}, B^{\prime} \subset V$ and an $\operatorname{arc} D$ satisfying the following conditions.

(i) $\quad A \supset A^{\prime} \supset M ; B \supset B^{\prime} \supset M ; e(D)=d\left(B^{\prime}\right)$;

(ii) $\quad D \cap\left(A^{\prime} \cup B^{\prime}\right)=e\left(B^{\prime}\right)$;

(iii) $A$ meets the interior of the closed curve $B^{\prime} \cup D$ in a point $a_{1}$ and the exterior at a point $a_{2}$.

Then we say that $A V$-crosses $B$. If also

$$
e\left(B^{\prime}\right) \cap A^{\prime}=\varnothing
$$

then we say that $A$ crosses $B$; and if in addition

$$
e\left(A^{\prime}\right) \cap B^{\prime}=\varnothing,
$$

we say that $A$ s-crosses $B$.

If $M$ consists of a single point, we speak of a point-crossing, point- $V$ crossing, or point-s-crossing.

Of these only the $s$-crossing is symmetric. An example of $A$ crossing $B$ but $B$ not crossing $A$ occurs when $A$ is the $x$-axis from -1 to 2, $M$ is the $x$-axis from 0 to 1 , and $B$ is $M$ and $y=x \sin 1 / x(-1 \leqq x<0)$.

Lemma 1. If $A$ s-crosses $B$ at $M$, then $B$ s-crosses $A$ at $M$.

Proof. Let $A^{\prime}, B^{\prime}, a_{1}, a_{2}, D$ be as in the definition. Join the end points of $A^{\prime}$ by an arc $D_{2}$ which meets $A^{\prime}$ nowhere else. Then $D_{2} \cup A^{\prime}$ is a simply closed curve. Now $B^{\prime} \cap D_{2} \cap A^{\prime}=\varnothing$. Hence there is a subarc $B_{1}$ of $B$ such that $M \subset B_{1}, B_{1} \cap D_{2}=\varnothing$, and $e\left(B_{1}\right) \cap A^{\prime}=\varnothing$. Such a subarc $B_{1}$ may be obtained by taking any subarc in the interior of the largest subarc $B_{3}$ of $B$ containing $M$ whose interior is disjoint from $D_{2}$, but itself containing in its interior the largest subarc of $B_{3}$ with end points in $A^{\prime}$.

If $B_{1}$ has points both interior and exterior to $D_{2} \cup A^{\prime}$, then $B$ $s$-crosses $A$ at $M$. Otherwise suppose that no point of $B_{1}$ is interior to $D_{2} \cup A^{\prime}$.

Choose a neighborhood of $M$ sufficiently small that its boundary meets $A^{\prime}$ in points separated by $M$ and likewise for $B^{\prime}$. In $W$ there are $\operatorname{arcs} A_{1}^{\prime}, B_{1}^{\prime}, D_{1}$ satisfying the definition of $s$-crossing. Necessarily $A_{1}^{\prime} \subset A^{\prime}, B_{1}^{\prime} \subset B^{\prime}$; let $a_{1}^{\prime}$ be a point of $A_{1}^{\prime}$ lying interior to $B_{1}^{\prime} \cup D_{1}$, 
and $a_{2}^{\prime}$ a point of $A_{1}^{\prime}$ exterior. Let $\epsilon$ be the smaller of the distance of $A_{1}^{\prime}$ from $D_{1}$ and of $a_{1}^{\prime}, a_{2}^{\prime}$ from $B_{1} \cup D_{1}$; thus $\epsilon>0$. Choose points $p_{1}, p_{2}$ interior to $D_{2} \cup A^{\prime}$ but with the distance of $p_{1}$ to $a_{1}^{\prime}$ and $p_{2}$ to $a_{2}^{\prime}$ less than $\epsilon$. Thus $p_{1}$ is interior to $B_{1}^{\prime} \cup D_{1}$ and $p_{2}$ exterior. Join $p_{1}$ to $p_{2}$ by an $\operatorname{arc} E$ lying interior to $D_{2} \cup A^{\prime}$ and every point of which is less than distance $\epsilon$ from $A_{1}^{\prime}$. Then $E$ does not intersect $D_{1}$, but has points interior and exterior to $D_{1} \cup B_{1}^{\prime}$. Hence it intersects $B_{1}^{\prime}$ and a fortiori $B^{\prime}$. But since $E$ lies interior to $D_{2} \cup A^{\prime}$ it cannot intersect $B^{\prime}$. This contradiction shows that the assumption that $B_{1}$ had no points interior to $D_{2} \cup A^{\prime}$ is false.

A very similar argument shows that we cannot assume that $B_{1}$ has no point exterior to $D_{2} \cup A^{\prime}$. Thus $B_{1}$ has points both interior and exterior to $D_{2} \cup A^{\prime}$. Hence $B_{1} s$-crosses $A$ at $M$.

Lemma 2. If $A$ does not cross $B$ at $M$, then $A V$-crosses $B$ at $M$ if and only if $A$ crosses $B$ in every neighborhood of $M$.

The proof for "if" is immediate.

Conversely, suppose $A V$-crosses, but does not cross, $B$ at $M$. Then for some neighborhood $V$ of $M$ the points $a_{1}, a_{2}$ as given in the definition of $V$-crossing will be the end points of a subarc $A_{1}$ of $A$ which does not contain $M$. In going from $a_{1}$ to $a_{2}$ on $A_{1}$ let $m$ be the last point which lies on $B$ such that no preceding point lies exterior to $B^{\prime} \cup D$, and let $M_{1}$ be the component of $A^{\prime} \cap B^{\prime}$ containing $m$. This component $M_{1}$ is contained in the interior of $B^{\prime}$ since $e\left(B^{\prime}\right) \cap A=\varnothing$. Also $M_{1}$ is contained in the interior of $A_{1}$ since it lies between $a_{1}$ and $a_{2}$ and does not contain them.

We show finally that $A$ crosses $B$ at $M_{1}$. Let $V_{1}$ be a neighborhood of $M_{1}$. By our construction the component of $B \cap V_{1}$ which contains $M_{1}$ has points $b_{1}, b_{2}$ separated by $M_{1}$ on $B$ and not in $A$. Let $B_{1}^{\prime}$ be the subarc of $B$ with end points $b_{1}, b_{2}$. Let $D_{1}$ be the rest of the simple closed curve $B \cup D$; then $B_{1} \cup D_{1}$ and $B \cup D$ are the same simple closed curves. Let $r_{1}$ be the distance of $M_{1}$ from $D_{1}$; hence $r_{1}>0$. Let $V$ be the set of points whose distance from $M_{1}$ is less than $r_{1}$, and let $A_{1}^{\prime}$ be the component of $M_{1}$ in $A \cap \bar{V}$ ( $\bar{V}$ denotes the closure of $V$ ). Then by the construction $A^{\prime}$ contains points $a_{1}^{\prime}, a_{2}^{\prime}$ separated by $M_{1}$ and not in $B$, where $a_{2}^{\prime}$ is exterior to $B \cup D$ and $a_{1}^{\prime}$ is of necessity interior. Then in the definition of $A$ crossing $B$ at $M_{1}$ use $V_{1}, A_{1}^{\prime}, B^{\prime}$, $a_{1}^{\prime}, a_{2}^{\prime}$.

Theorem 1. If an arc $A$ crosses an arc $B$, then $B-A$ contains $a$ neighborhood of the origin.

Let $A^{\prime}, B^{\prime}, D, a_{1}$, and $a_{2}$ be as described in the definition. Let $A^{\prime \prime}$ 
be the subarc of $A^{\prime}$ joining $a_{1}$ and $a_{2}$. Let $r$ be the smallest of the following distances: of $a_{1}$ from $B^{\prime} \cup D$, of $a_{2}$ from $B^{\prime} \cup D$, and of $A^{\prime \prime}$ from $D$. Translate $A^{\prime \prime}$ by an amount $r^{\prime}<r$ in any direction $\theta$ to obtain an $\operatorname{arc} A_{1}$; then $A_{1}$ cannot meet $D$. Now $a_{1}$ goes to a point $a_{1}^{\prime}$ and $a_{2}$ to $a_{2}^{\prime}$; also $a_{1}^{\prime}$ is interior to $B^{\prime} \cup D$ while $a_{2}^{\prime}$ is exterior. Hence $A_{1}$ intersects $B^{\prime} \cup D$ and must do so in $B^{\prime}$ and at a point $b$ which is the image under the translation of a point $a$ of $A$. The point $b-a$ has polar coordinates $\left(r^{\prime}, \theta\right)$. Thus $B-A$ contains the interior of the circle of radius $r$ and with center at the origin.

The converse of the theorem is not true. As a counterexample let $A$ and $B$ both be the spiral given in polar coordinates by $r=1 / \theta$ $(1 \leqq \theta \leqq \infty)$ and $M$ be the origin. Another counterexample is given by letting $A$ be the double spiral $r=1 / \theta$ and $r=1 /(\theta+\pi / 2)$ and $B$ the double spiral $r=1 /(\theta+\pi)$ and $r=1 /(\theta+3 \pi / 2)$, where throughout $1 \leqq \theta \leqq \infty$.

Yet another counterexample occurs when $A$ and $B$ are the same triod $S$; i.e., $S$ consists of three arcs, having an end point $p$ in common but no other point common to any two of them.

LEMмA 3. If $S$ is a triod then $E(S)$ contains a neighborhood of the origin.

Suppose that $S$ consists of the three $\operatorname{arcs} A, B, C$, each having $p$ as an end point and no two having any other point in common. Let the other end points be $a$ on $A, b$ on $B$, and $c$ on $C$. Let $D$ be a simple closed curve containing $a, b, c$ but no other points of $A, B, C$, and with $p$ inside $D$. Let $D_{a b}$ be the subarc of $D$, with end points $a$ and $b$ and not containing $c ; D_{b c}$ and $D_{c a}$ are similarly defined. Then $D=D_{a b}$ $\cup D_{b c} \cup D_{c a}$. Also $D_{a b} \cup A \cup B$ is a simple closed curve.

The remainder of the proof is similar to that of Theorem 1. Let $a^{\prime}$ be a point on the interior of $A$ and let $A^{\prime}$ be the subarc of $A$ joining $p$ and $a^{\prime}$. Define $b^{\prime}, B^{\prime}$ and $c^{\prime}, C^{\prime}$ similarly. Let $r_{a}$ be the lesser of the distances of $a^{\prime}$ from $B \cup C$ and $A^{\prime}$ from $D$, with corresponding definitions for $r_{b}$ and $r_{c}$. Take $r$ as the minimum of $r_{a}, r_{b}, r_{c}$. Then $r>0$.

Translate $F=A^{\prime} \cup B^{\prime} \cup C^{\prime}$ by an amount $r^{\prime}<r$ in any direction $\theta$, obtaining the set $F_{1}$. Then $p$ goes into a point $p_{1}$ which must lie inside $D$. But $p_{1}$ must be inside or on the boundary of one of the simple closed curves $D_{a b} \cup A \cup B, D_{b c} \cup B \cup C$, or $D_{c a} \cup C \cup A$; assume it is so for the first curve. Then the image $c_{1}^{\prime}$ of $c^{\prime}$ lies outside that simple closed curve and the image $C_{1}^{\prime}$ of $C^{\prime}$, having one end point outside and other inside or on the boundary of $D_{a b} \cup A \cup B$, must intersect it at a point $w$ which is also the image $z_{1}$ of a point $z$ on $C^{\prime}$. This intersection $w$ cannot be on $D$; hence it lies on $A \cup B$. Thus $w-z=z_{1}-z$ 
is the point with polar coordinates $\left(r^{\prime}, \theta\right)$ and lies in $E(S)$. This shows that $E(S)$ contains the circle of radius $r$ and center at 0 , as was to be proved.

Theorem 2. Let $B$ be an arc for which $E(B)$ has no point with vectorial angle $\theta$ except the origin, i.e., no secant of $B$ has inclination $\theta$. Suppose $M$ is a subarc in the interior of $B$ and also of an arc $A$. Then $A$ does not cross $B$ at $M$ if and only if there are a neighborhood $V$ containing $M$ and $a$ direction $\alpha$ such that no point in $(B \cap V)-(A \cap V)$ has vectorial angle $\alpha$ except the origin.

The "if" part is a consequence of the previous theorem.

If $A$ does not cross $B$ at $M$ there is a subarc $A^{\prime}$ of $A$ and a subarc $B^{\prime}$ of $B$, both containing $M$ but with end points different from those of $M$, and an arc $D$ joining the end points of $B^{\prime}$ and not otherwise intersecting $A^{\prime}$ or $B^{\prime}$ such that no point of $A^{\prime}$ lies in the interior of the simple closed curve $B^{\prime} \cup D$. Choose a subarc $B^{\prime \prime}$ of $B^{\prime}$ containing $M$ but with end points distinct from those of $B^{\prime}$. Let $d$ be the distance of $B^{\prime \prime}$ from $D$. Let $W$ be the set of points with polar coordinates $\left(d^{\prime}, \theta\right), 0<d^{\prime}<d / 2$. None of the points of $B^{\prime \prime}+W$ lies on $D$ since $d^{\prime}$ $<d / 2$ and none lies on $B$ by the hypothesis on $\theta$. The same is true of $B^{\prime \prime}-W$. Thus either $B+W_{1}$ or $B-W_{1}$ lies in the interior of $B^{\prime} \cup D$; let it be denoted by $B+W$. Let $\alpha=\theta+\pi$ or $\theta$ according as $W=W_{1}$ or $-W_{1}$. Then $B^{\prime \prime}-W$ lies interior to $B^{\prime} \cup D$ and hence cannot intersect $A^{\prime}$. Thus $B^{\prime \prime}-A^{\prime}$ does not contain $W$. Finally, if $V$ is taken such that its distance from $M$ is $<d / 2$, we see that the theorem is satisfied.

2. Local boundary point. Before returning to the problem of lattice packings, we introduce another term.

Definition. A point $p$ is called a local boundary point of $A+B$ if (1) $p$ lies in the closure $\mathrm{Cl}(A+B)$ of $A+B$, and (2) whenever $p=a+b$ with $a$ in $A$ and $b$ in $B$, there exist neighborhoods $U$ of $a$ and $V$ of $b$ such that $p$ is a boundary point of $(A \cap U)+(B \cap V)$.

By a local boundary point of $E(S)$ is meant a local boundary point of $S+(-S)$.

If $S$ is composed of three sides of a square, then $E(S)$ is a square and the locus of its local boundary points consists of the four sides and also the two lines joining the midpoints of opposite sides.

LEMMA 4. If $A$ is open, then $A+B$ contains none of its local boundary points.

For if $p=a+b$ with $a$ in $A$ and $b$ in $B$, then for every neighborhood $U$ of $a$ with $U \subset A, U+b$ is a neighborhood of $p$ and $U+b \subset A+B$. 
Hence for any neighborhood $V$ of $b,(U \cap A)+(V \cap B)=U+(V \cap B)$ contains the neighborhood $U+b$ of $p$ since $b$ is in $V \cap B$. Thus $p$ cannot be a local boundary point.

The converse, that if $A+B$ contains none of its local boundary points then either $A$ or $B$ is open, is not true. Counterexample: $A=B$ and is the punctured disc consisting of every point whose distance $d$ from the origin satisfies $1 \leqq d<4$.

\section{Lattice packings.}

Theorem 3. In order that $S+\Lambda$ be a lattice packing without pointcrossing arcs it is sufficient that the points of $\Lambda$ distinct from 0 and belonging to $E(S)$ be local boundary points of $E(S)$.

The proof is by contradiction. Suppose that there are $\operatorname{arcs} A$ and $B$ in $S$ such that $A+\lambda^{\prime}$ crosses $B+\lambda^{\prime \prime}$, where $\lambda^{\prime}$ and $\lambda^{\prime \prime}$ are distinct points of $\Lambda$. Then $A$ and $B+\lambda$ cross, where $\lambda=\lambda^{\prime \prime}-\lambda^{\prime}$. Hence by Theorem 1 , $A-(B+\lambda)$ contains a neighborhood of 0 and $A$ and $B+\lambda$ may be be taken to lie in any neighborhood, no matter how small, of the point of crossing. Thus $A-B$ contains a neighborhood of $\lambda$. Also $A-B$ lies in $E(S)$. Hence $\lambda$ is not a local boundary point of $E(S)$. But $\lambda$ is a point of $\Lambda$ distinct from 0 . The existence of such a point is a contradiction to the hypothesis on the nonexistence of such points, because of Lemma 2.

If in Theorem 3 crossings on common subarcs, not simply points, are to be excluded, then the following modification needs to be made. Whenever two subarcs $A, B$ of $S$ can be made to coincide by a translation, i.e., $A=B+p$ for some point $p$, then in determining whether $p$ or $-p$ is a local boundary point only those neighborhoods of $A$ or $B$ are used which include maximal arcs $A_{1}$ or $B_{1}$ such that $A_{1} \subset A, B_{1} \subset B$, and $A_{1}=B_{1}+p$.

Although Theorem 2 provides a partial converse to Theorem 1, yet a partial converse of Theorem 3 cannot be obtained from Theorem 2 without imposing rather stringent hypotheses.

An example illustrating some of the difficulty is the following. On the interval $-\epsilon \leqq x \leqq \epsilon$, let $S$ be the set of all horizontal line segments with $y$ rational and between $-\epsilon$ and $\epsilon$ and let $T$ be the same except that $y$ is irrational or 0 . Then $S-\Gamma$ contains a neighborhood of the origin and the origin is not a local boundary point, although $S$ and $T$ do not have crossing arcs.

Theorem 2 could be used if one required that on every $\operatorname{arc} A$ of $S$ every point has a neighborhood in which the secants of $A$ do not have all directions and by modifying the definition of a local boundary 
point so that instead of $U \cap A$ and $V \cap B$ one takes any subarc of $A$ in $U$ containing $a$ and any subarc of $B$ in $V$ containing $b$. These changes are felt to be undesirable in being too great a departure from the original concept of $D(S)$ and in excluding sets with nonempty interiors.

UNIVERSITY OF MichIGAN

\title{
THE DEGREE FORMULA FOR THE SKEW-REPRESENTA- TIONS OF THE SYMMETRIC GROUP1
}

\author{
W. FEIT
}

1. Introduction. In his paper on the representations of the symmetric group, ${ }^{2}$ G. de B. Robinson defines certain "skew-representations" and associates these to skew-diagrams (to be defined below) analogously to the way the irreducible representations of the symmetric group are associated with regular diagrams. Furthermore he shows that the degree of such a skew-representation is equal to the number of orderings of the related skew-diagram. ${ }^{3}$

The object of this note is to derive a formula for the degree of skewrepresentation related to a given skew-diagram. ${ }^{4}$ This problem will be treated strictly in terms of the number of orderings of such a diagram, and from this point of view is very similar to the question attacked in [5] by R. M. Thrall.

In $\S 4$, this formula is applied to the problem of computing the characters of certain classes of the symmetric group.

2. Definitions and lemmas. A partially ordered set $P$ is said to be regular or a regular diagram if:

(I) The elements of $P$ may be represented by ordered pairs of integers $(i, j), i>0, j>0$, where $(i, j) \leqq(p, m)$ if and only if $i \leqq p$ and $j \leqq m,(i, j)=(p, m)$ if and only if $i=p$ and $j=m$,

(II) $\max _{i}(i, j) \leqq \max _{i}\left(i, j^{\prime}\right)$ whenever $j \geqq j^{\prime}$,

(III) $(i, k) \in P$ implies $(j, k) \in P$ for all integers $j$ with $1 \leqq j \leqq i$,

Presented to the Society, April 25, 1952; received by the editors March 9, 1953.

1 The work on this paper was performed under the sponsorship of the O.N.R.

2 See $[1 ; 2 ; 3 ; 4]$.

${ }^{3}$ See $[1$, p. 290].

4 This is an answer to the question raised in $[1$, p. 294], the $\phi$ of that paper is the $g$ of the theorem below. 